\title{
Conjugate and Disjunctive Saccades in Two Avian Species with Contrasting Oculomotor Strategies ${ }^{1}$
}

\author{
JOSH WALLMAN ${ }^{\star 2}$ AND JOHN D. PETTIGREW $\ddagger^{3}$ \\ *Department of Biology, City College of City University of New York, New York, New York 10031, and $\ddagger$ National Vision Research \\ Instilute of Australia, 386 Cardigan Street, Carlton 3052, Australia
}

\begin{abstract}
We have recorded with the magnetic search coil method the spontaneous saccades of two species of predatory birds, which differ in the relative importance of panoramic and foveal vision. The little eagle (Haliaetus morphnoides) hunts from great heights and has no predators, whereas the tawny frogmouth (Podargus strigoides) hunts from perches near the ground, is preyed upon, and frequently adopts an immobile camouflage posture.

We find that both birds spend most of the time with their eyes confined to a small region of gaze, the primary position of gaze; in this position, the visual axes are much more diverged in the frogmouth than in the eagle, thereby giving it a larger total field of view at the expense of its binocular field. Both species, however, can align their visual axes, thereby gaining a binocular view of targets in the frontal visual field. The eagle makes saccades 10 times as frequently as the frogmouth, and in most of its saccades the eyes move in the same direction, although the eye movements are generally not conjugate; in most frogmouth saccades, on the other hand, the eyes move in opposite directions. We discuss these phenomena in terms of their saccadic mechanisms and their possible adaptiveness. We also report two incidental observations: in certain circumstances, the frogmouth shows no sign of having a vestibulo-ocular reflex, and the eagle, although the quintessential foveate animal, shows extreme directional asymmetries of monocularly evoked optokinetic nystagmus.
\end{abstract}

Although much is known about the mechanism of production of saccadic eye movements and of the details of saccadic trajectories, little is known about what determines the timing, direction, and amplitudes of saccades produced in normal life. One possibility is that all of the thousands of saccades made daily are directed at particular targets. Alternatively, it may be that in the absence of specific targets, saccades are made nonetheless with some statistically definable pattern of directions, amplitudes, and intersaccadic

Received July 13, 1984; Revised December 18, 1984

Accepted January 2, 1985

' This work was supported by National Institutes of Health Grant EY02937 to J. W. and grants from the Australian National Health and Medical Research Council to J. D. P.

${ }^{2}$ To whom correspondence should be addressed, at Department of Biology, City College of City University of New York, Convent Avenue and 138th Street, New York, NY 10031.

${ }^{3}$ Present address: Department of Physiology, University of Queensland, St. Lucia, QLD 4067, Australia. intervals. We hypothesize that saccades have such an endogenous pattern in time and space, and that this pattern represents an oculomotor strategy for a species.

As a start in investigating this hypothesis, we have taken advantage of the diversity of avian visual adaptations by studying spontaneous saccades made by two species, the tawny frogmouth and the little eagle, which we presume have different oculomotor requirements, particularly with respect to binocular coordination. The saccades of both eyes were studied as the animals freely viewed the laboratory with their heads restrained. Although this situation is of coursc far from what the animals would experience in their natural circumstances, it provides an opportunity to compare the two species under identical conditions without the complication of differences in head movements. To aid in the interpretation of differences in oculomotor behavior in the two species, we will briefly describe their natural history.

The tawny frogmouth (Podargus strigoides) is a representative of a Gondwanaland group of Caprimulgiformes, which are close relatives of the owls (Fig. 1). The following account of their habits is taken from Schodde and Mason (1981). Like owls, the frogmouth is a nocturnal predator, feeding on invertebrates and small vertebrates which it takes mostly on the ground after short sallies from a low perch, although a small amount of aerial feeding also occurs. As a camouflage strategy, the frogmouth relies heavily on its cryptic plumage and a posture in which it resembles a broken branch. This camouflage strategy is sufficiently effective that observers can walk close to a frogmouth in daylight without recognizing it. Nonetheless, frogmouths have been found to be preyed upon by hawks and owls. When adopting the "camouflage posture," a frogmouth becomes immobile and nearly closes its eyes, thereby masking its salient, bright yeltow irides, although it is possible at times to see eye movements behind the partially closed lids.

Frogmouths have a single fovea in the temporal retina of each eye. Since this fovea views the frontal visual field, it can be used in cooperation with that of the other eye for binocular vision and consequently is frequently referred to as the "binocular" fovea (Bravo and Pettigrew, 1981; Pettigrew, 1979).

The little eagle (Haliaetus morphnoides) is a small, but otherwise typical, eagle of the Accipitridae family of diurnal predators. The following details of the natural history of Haliaetus are from Cramp and Simmons (1981). Like other eagles, the little eagle hunts during the day by soaring aloft where it has a commanding view of the open or semiopen countryside over which it hunts. Prey are taken on the ground after a long dive or, more rarely, in the air. Apart from humans, an adult little eagle has no significant predator. Like other diurnal raptors, eagles have two foveas in each retina, a "binocular fovea" in the temporal retina, and a "monocular fovea" near the optic axis (Wood, 1917).

The evolution of the visual system in all species involves a tradeoff between the advantages of a maximally large visual field for panoramic vision and those of a large area of overlap of the two 

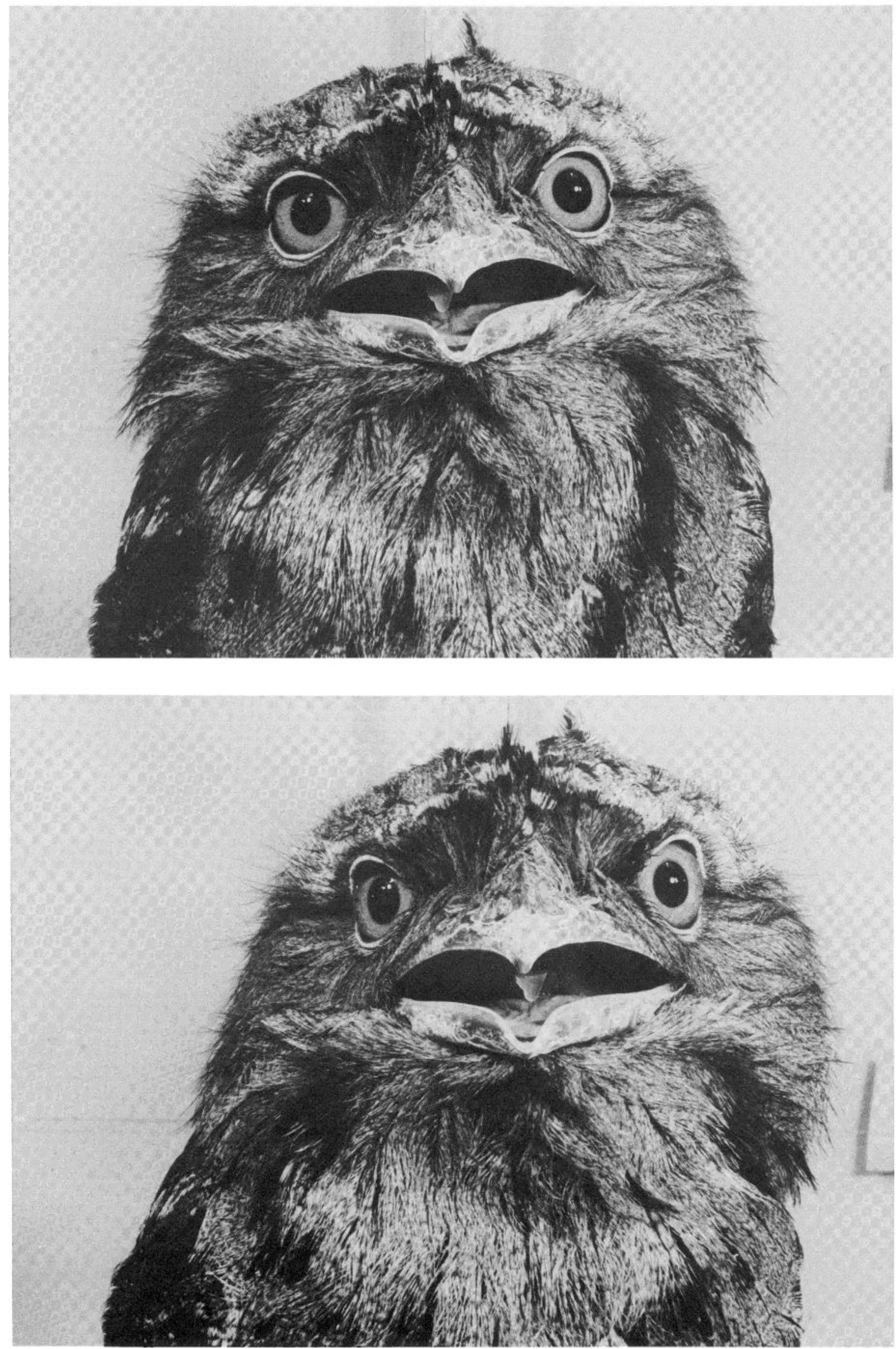

Figure 1. Photographs of tawny frogmouth, showing two characteristic states of vergence of the eyes. In the upper photograph, both eyes are frontally directed. The convergent posture was induced by presenting a mouse in the frontal field of this tame bird. (Wild frogmouths tend to adopt a divergent posture in the presence of humans.) Note that the corneal reflections of the lights are near the center of the pupil. In the lower photograph, the eyes are in the lateral position of gaze, as is evident from the nasal position of the corneal reflections. This divergent posture was induced by a loud clap. 
visual fields for binocular vision. We would expect panoramic vision to be less important for the eagle than for the frogmouth for at least two reasons. First, frogmouths are sometimes taken as prey themselves and might benefit particularly from panoramic vision especially when sitting immobile in the camouflage posture. Second, since frogmouths hunt close to the ground, the location of potential prey items would therefore cover a wider angular substense than would be the case for an eagle soaring at a great height. Consequently, the competing demands of panoramic and binocular vision, in addition to the presence of the bifoveate specialization in one but not the other species, might be expected to result in different oculomotor strategies for each species. We have therefore documented the patterns of horizontal eye movements of these species, with a particular emphasis on binocular coordination. These patterns not only differ between the two species studied, but also differ from the well-described, highly conjugate eye movements of mammals.

\section{Materials and Methods}

\section{Subjects, experimental conditions, and recording methods}

The results presented here are based on measurements using the mag. netic search coil technique to examine the spontaneous eye movements of two adult tawny frogmouths and one little eagle. The subjects used were available because because two had injuries that precluded their rehabilitation to the wild, while the other was a hand-raised very tame bird. To record eye movements, each bird was placed in a cubical box, $65 \mathrm{~cm}$ square, made of transparent acrylic plastic, around which were two acrylic frames onto which the field coils were wound. The bird's body was wrapped in a towel and placed in a cradle so that the eyes were in the geometric center of the coils. To prevent head movements, the beak was cemented to a plastic bar in such a manner that it could readily and painlessly be released. The bird was then left alone in the room during the recording. Several such episodes make up the data for the present analysis. In a fow scgments of the records, drifting eye movements or nystagmus was seen in both eyes. This was taken as evidence of lack of alertness and these records were not analyzed. Since our concern was principally with binocular coordination of the eyes, we studied only horizontal eye movements. Although it would have been desirable to record the eye movements in three orthogonal planes, the technical ditticulties of recording torsional eye movements deterred us. ${ }^{4}$

The field coils were energized at $25 \mathrm{kHz}$ by a conventional oscillator and audio amplifier. The signal from the eye coils went to an amplifier/phase detector (Evans Associates, Berkeley, CA) with a DC-500 Hz frequency response and then to an oscilloscope and chart recorder. Measurement of the saccades from the chart records was done with a digitizing tablet connected to a computer. Both the time and the position of both eyes before and after each saccade were measured. Because we were recording spontaneous eye movements and the birds had substantial oculomotor ranges, we recorded at relatively low sensitivity. Consequently, very small saccades (less than $1^{\circ}$ ) could not be measured very accurately. Instrument noise and drift were not present at this sensitivity. The time resolution of this method was approximately $10 \mathrm{msec}$ in the high-speed records, and 1 to 3 sec in the low-speed records used for studying the frequency and amplitude distributions of saccades.

\section{Coil implantation}

Both eyes of each animal were implanted with scleral search coils. Anaesthesia was induced with a mixture of ketamine $(10 \mathrm{mg}$ intramuscularly

\footnotetext{
${ }^{4}$ Although in humans and other frontal-eyed animals, it is perhaps reasonable to assume that the movements in the horizontal and vertical planes are the most important ones, and that the movements in the torsional plane can be ignored, this assumption is demonstrably untrue in more lateral-eyed animals (Collewijn and Noorduin, 1972; Lanchester and Mark, 1975; Wallman and Velez, 1985). For example, in the animals studied here, in which the optic axes of the eyes are approximlately $45^{\circ}$ to the sagittal plane, following an object moving vertically in front of the animal would involve eye movements with equal components in the vertical and torsional planes. Since without measuring all three planes of eye movement we could not assess the actual alignment of the foveas or the true saccade amplitudes or velocities, we have confined our analysis to measures available from the horizontal records.
}

(i.m.) for the frogmouths and $1.5 \mathrm{mg}$ i.m. for the eagle) and xylazine (1 $\mathrm{mg}$ i.m. for the frogmouth and $1.5 \mathrm{mg}$ i.m. for the eagle) and then continued with 0.25 to $0.5 \%$ halothane in $25 \%$ nitrous oxide/ $75 \%$ oxygen delivered via a T. piece to an endotracheal cannula. The method of coil implantation was a combination of two conventional methods (Fuchs and Robinson, 1966; Judge et al., 1980). Teflon-coated, 14-stranded stainless steel wire (AS632 Cooner Wire Co., Chatsworth, CA) was threaded bolow the conjunctiva 2 to $3 \mathrm{~mm}$ beyond the limbus through two or three holes with an aneurysm needle fashioned from a darning needle so that its radius of curvature was appropriate for the eye in question ( $6 \mathrm{~mm}$ for the eagle, $10 \mathrm{~mm}$ for the frogmouth). The ends of the wires were twisted together and formed into a take-up loop placed in a pocket dissected from the connective tissue of the orbit and then passed with a needle through the tendinous, posterolateral roof of the orbit to connect to a plug which was cemented to the skull and to two small screws in the skull. In both birds, the eyes looked normal immediately after the implantation operation, and appeared to have a full range of movements of globe, nictitans, and eyelids. In the frogmouth, the lid's of one eye tended to be held partly closed for a week after the surgery.

\section{Retinal landmarks by ophthalmoscopy}

Repeated determinations of the projections of retinal landmarks show great reproducibility in anaesthesized birds of the same species (Pettigrew, unpublished data). We therefore determined the interocular and intraocular relationships of the identifiable retinal landmarks (pecten, binocular fovea, monocular fovea, and ora terminalis) in both birds, anaesthesized before and after the coil implantation. Anesthesia and muscle relaxation were obtained as described above. The beak was fixed to a horizontal bar, and retinal landmarks were identified with a ophthalmoscope mounted on a flexible toolholder and fitted with a corner-cube prism in a slide. When the retinal landmark of interest had been identified and centered in the target graticle of the ophthalmoscope, the corner-cube could be slid into place to reflect the target onto a tangent screen. In the frogmouth, the binocular fovea was discernible as a shallow depression in the temporal retina, on a line extending roughly from the superior tip of the pecten and parallel with its long axis. The fovea was obvious by a change in the bluish specular reflection from the surface of the retina, which tends to break into an arc near the fovea. In the eagle, both binocular and monocular foveas were readily discernible as dark pits lying at the center of two brilliant blue, circular reflections.

In both species, the edge of the temporal retina appears as a sudden transition from the pink brightly reflective retinal surface to the completely black pigment of the ora terminalis. Since this defines the theoretical limit of vision in that direction for each eye, plotting the projection of the ora terminalis into space permits a measure of the possible degree of binocular overlap, by doubling the horizontal separation of the ora terminalis from the binocular fovea; this assumes that the maximal useful binocular overlap occurs when the binocular foveas are aligned. The estimations were carried out in the first $30 \mathrm{~min}$ after injection of ketamine and xylazine, during which no eye movements were detected by use of the ophthalmoscope; $45 \mathrm{~min}$ after the iritial anlaesthesia, small drifts were sometimes observed.

\section{Calibration}

We used two methods to establish the relationship between the direction of the visual axis given by the binocular fovea and the signal coming from the coil on that eye. The method used for the eagle was to have onc obscrver locate the pecten tip with an ophthalmoscope, at which time the other observer both marked the point at which the ophthalmoscope beam passed through the transparent plexiglass enclosure surrounding the bird and simultaneously marked the corresponding position of the eye on the chart recorder. The ophthaimoscope was then moved to one side by approximately $10^{\circ}$, and the observer waited until the eagle looked in such a direction as to again line up the pecten tip with the ophthalmoscope; then the procedure was repeated. During the brief period of these calibrations, the eagle did not seem to avoid the ophthalmoscope, perhaps because the pecten represents a blind spot on the retina. After the calibration trials, the solid angles between the loci marked on the enclosure were calculated.

We could not use this calibration technique with the frogmouth, since it suppressed nearly all eye movements when being examined with an ophthalmoscope. Instead, we took advantage of this ocular immobility (which included essentially complete loss of the vestibulo-ocular reflex-see "Results") and rotated the bird by $10^{\circ}$ about an axis passing between the eyes. We confirmed the extent of ocular rotation by having an observer with an ophthalmoscope positioned $10^{\circ}$ from the starting point. We then measured the deflection on the chart recorder caused by this rotation.

In addition to determining the relationship between the chart recorder trace and the direction in space in which the fovea was looking, it was also 


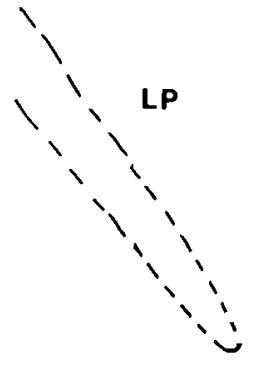

LMF $\because \because \because ;$

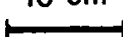

LITTLE EAGLE<smiles>CCC[Pb]</smiles>

TAWNY FROGMOUTH

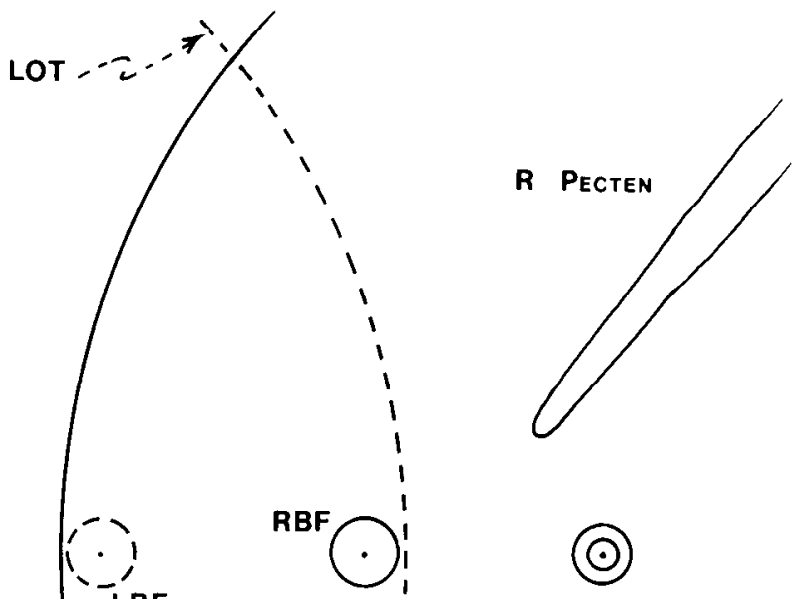

R Monocular Fovea

R Ora Terminalis

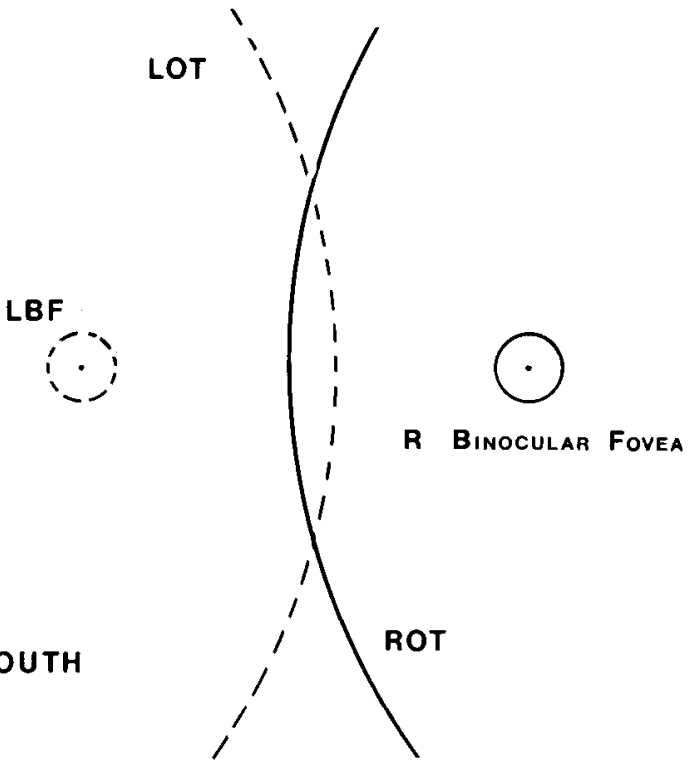

necessary to estimate the angular position of the coil on the eye in order to correct for the sinusoidal distortion that exists, because the search coil technique produces signals proportional to the sine of the coil angle. In the case of the eagle, we could inter the angle of the optic axis from the retinal landmarks, since the monocular fovea, which lies close to the optic axis, was observed during calibration; we assumed that the orientation of the coil on the eye was $90^{\circ}$ to the optic axis. In the frogmouth, which lacks a monocular fovea, we made use of the arcsine relation between the response of the coil system and the eye position to infer the actual angle of the eye coil with respect to the external magnetic field. To do this, we plotted the eye-coil voltage at several calibration positions covering $40^{\circ}$. From this curve, we estimated that eye position from which deviations of the eye in both directions produced equal deviations in coil signal. In this position, the coil was perpendicular to the field coils.

\section{Results}

\section{Ophthalmoscopy}

In the presence of anaesthesia and muscle relaxation, both species had divergent visual axes. The angle subtended by the binocular foveas in the two frogmouths was $50^{\circ}$ and $52^{\circ}$, respectively, while for the eagle it was $24^{\circ}$. This is shown in Figure 2, which also shows the relationship between the other landmarks of each eye. It is of interest that the degree of binocular overlap which would be achieved if both binocular foveas were aligned is comparable in both species, although the frogmouth would have to make a much larger convergent movement from the position assumed during 
a

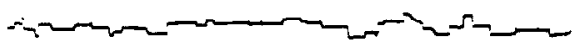

b

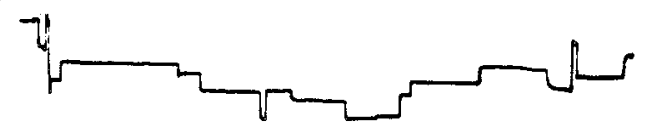

7

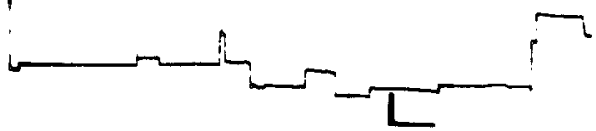

C

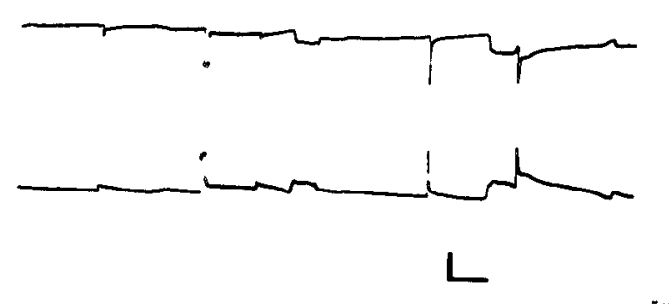

f

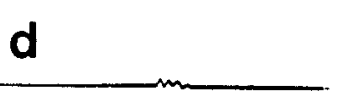

e
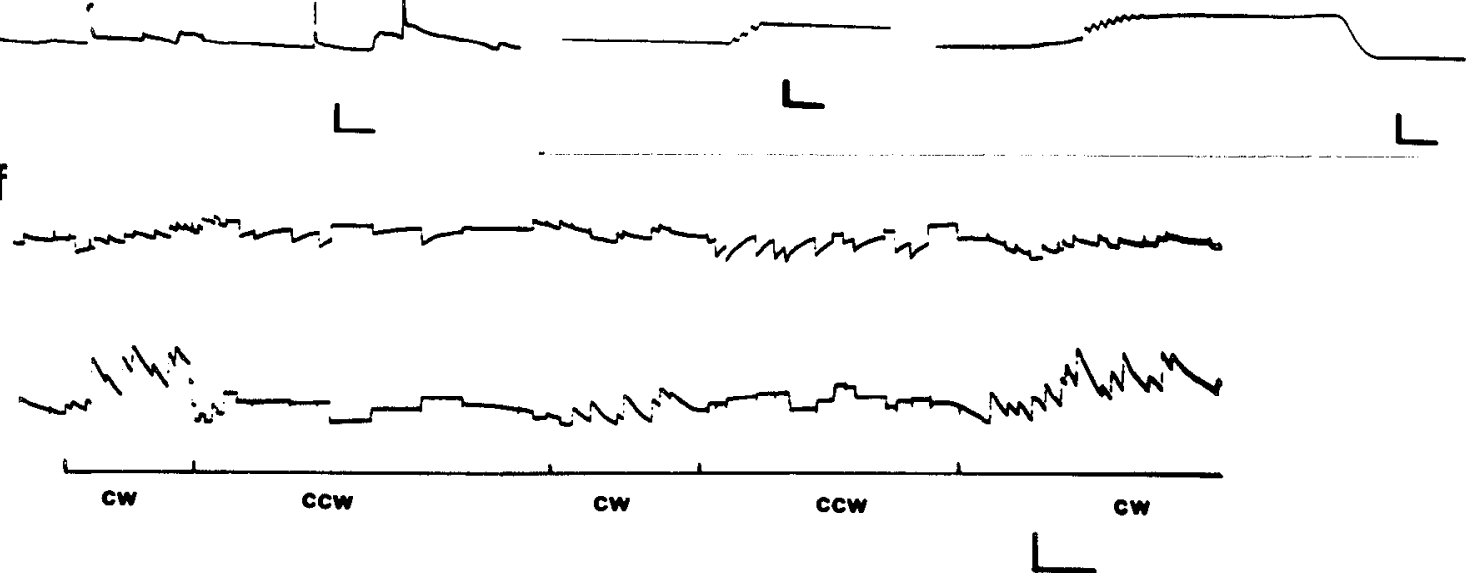

Figure 3. Raw eye movement records. $A$ to $C$, spontaneous saccades in eagle $(A)$ and frogmouth $(B$ and $C)$. Note difference in saccade frequency between species, and presence of brief episodes of convergent gaze in frogmouth. $D$ and $E$, individual saccades in eagle $(D)$ and frogmouth $(F)$, recorded at fast chart speed. Note relatively slow eye velocity in both species and presence of oscillations accompanying saccade. $F$, optokinetic nystagmus, monocularly evoked from the left eye of the little eagle. Note the extreme asymmetry of the OKN in the two directions of stimulus motion, with much highervelocity slow phases when the stimulus motion is in the temporal-to-nasal direction. In all records, eye positions to the right are plotted upward and the upper trace is the right eye. Vertical calibration marks are $10^{\circ}$. Horizontal calibration marks are as follows: $A, 10 \mathrm{sec} ; B, 20 \mathrm{sec} ; C, 40 \mathrm{sec} D D$ and $E, 0.2$ sec; $F, 10$ sec.

anesthesia than would the eagle, in which the binocular foveas straddle the region of binocular overlap.

\section{General characteristics of saccades}

Figure 3 shows sample records from each animal. Before discussing the quantitative differences between the saccades of each species, some general observations are in order.

1. Saccades in the two eyes are not yoked. Figure 3 shows clearly that in both animals saccades can be either conjugate or very different in amplitude or opposite in direction. Frequently, large saccades in one eye are accompanied by barely detectable saccades in the other.

2. Saccades in the two eyes are simultaneous. In both species, saccades in one eye were apparently always accompanied by a saccade in the other eye, even though one saccade may be very small. The limited dynamic range of the recording system prevents us from saying absolutely whether very small monocular saccades ever occur, but all measured saccades occurred synchronously in the two eyes in both species.

3. Saccades are slower than mammalian saccades and contain oscillatory eye movements. Figure $3, d$ and $e$, shows typical saccades of each species. A human saccade of the same amplitude would last approximately $40 \mathrm{msec}$ instead of the $250 \mathrm{msec}$ shown here. These oscillations are a general feature of avian saccades, previously reported in pigeons (Nye, 1969), owls (Steinbach and Money, 1973), and chickens (Turkel and Wallman, 1977), although these authors did not describe them as consistent saccadic components. The present authors have recorded similar oscillations accompanying the saccades of falcons, owls, and kookaburras.

\section{Primary position of gaze}

Examination of the raw eye movement records of both species showed that there was a position which the eyes tended to occupy much of the time and to which the eyes tended to return after saccadic excursions. To quantitate this observation, we constructed the dwell-time histograms of Figure 4. To do this, we took the average eye position (specifically the position of the binocular foveas) with respect to straight ahead during each intersaccadic interval, and plotted the number of seconds it remained there. It is clear from the figure that there is in both species a distinct modal eye position. We will refer to this position as the primary position of gaze. During $68 \%$ of the observation period (one SD on the dwell-time histogram), the cyos were within about $4^{\circ}$ (in the horizontal planc) of the mean eye position for both the eagle $\left(3.9^{\circ}\right)$ and the frogmouth $\left(4.1^{\circ}\right)$. It is of interest that despite the considerable difference between the two species in the range of horizontal movements recorded (frogmouth, $38^{\circ}$; eagle, $24^{\circ}$ ), there is so little difference in the range of positions in which the eyes spend most of their time. This argues for a distinct mechanism functioning either to return the eyes to the primary position of gaze or to keep the eyes near the primary position of gaze as opposed to this concentration of gaze near one position being a passive result of the spatial distribution of saccadic targets. 


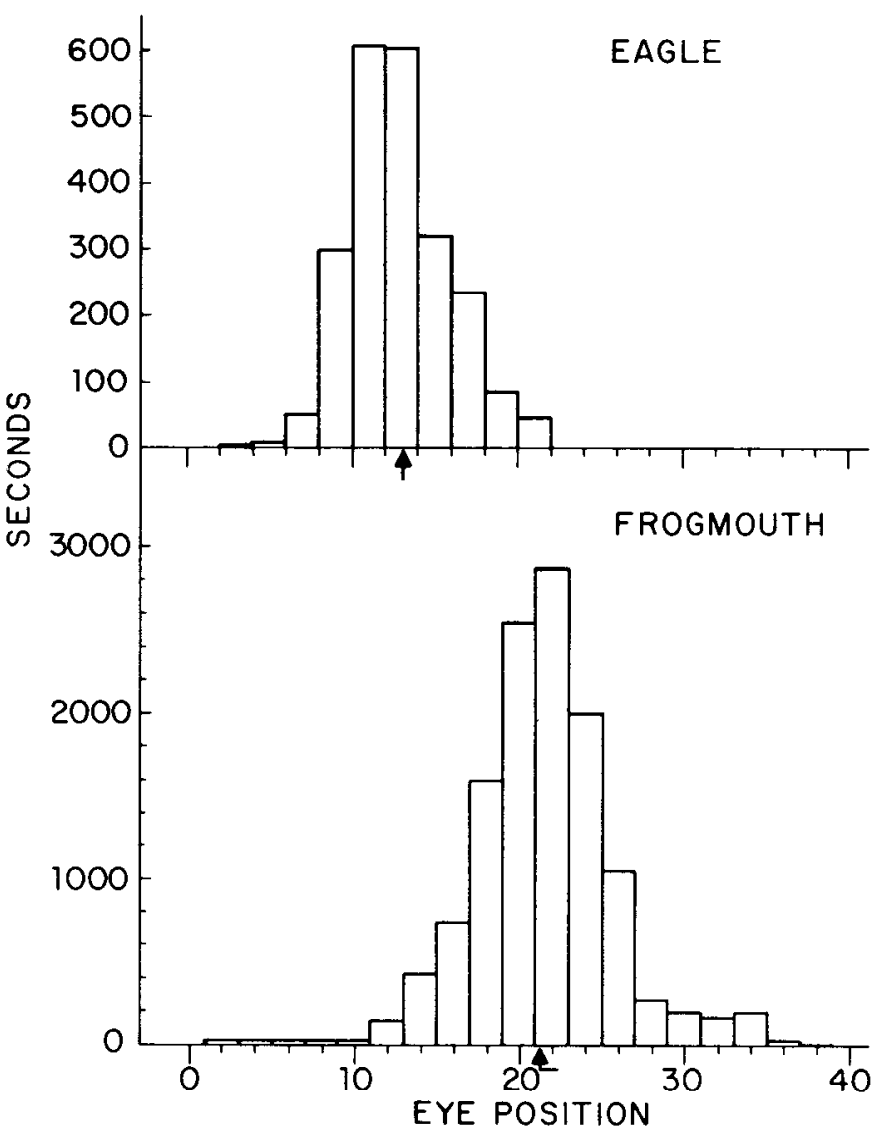

(GAZE ANGLE OF BINOCULAR FOVEA)

Figure 4. Dwell-time histograms showing amount of time eye spends at different horizontal position. Eye position is plotted as the angle (in degrees of $\operatorname{arc}$ ) between the line of gaze of the binocular fovea and the animal's midsagittal plane. Note that both species show a marked clustering about the mean position (arrow), although this position is more divergent in the frogmouth than in the eagle.

In the unrestrained rabbit, a similar, but broader, dwell time histogram has been found (Collewijn, 1977).

The location of the position of primary gaze differed in the two species in a way consistent with their differing needs for stereoscopic versus panoramic vision. In the eagle, the binocular foveas had a mean position $13^{\circ}$ from the sagittal plane; this placed them just within the binocular visual field, with the result that in the primary position of gaze the eyes were as divergent as possible without the foveas being in the monocular visual field. In contrast, in the frogmouth the eyes in the primary position are considerably more divergent, cach fovca bcing $21.5^{\circ}$ from the sagittal plane, yielding a greater total visual field at the expense of having almost no binocular field. In both species, the primary positions obtained from the dwelltime histograms of Figure 4 were within $1^{\circ}$ of the primary positions obtained by ophthalmoscopy in anaesthetized animals.

\section{Frequency of saccades}

A salient difference between the oculomotor behavior of these species is that the eagle makes quite frequent saccades (mean interval, $2.5 \mathrm{sec}$ ), whereas the frogmouth holds its eye stationary for much longer periods at a time (mean intersaccadic interval, $40 \mathrm{sec}$ ), as shown in Figure 5. In fact, the means and medians shown here tend to understate the difference between the species, because of the peak of many very short intervals in the frogmouth. This peak arises in part from the behavior of both eyes making large convergent movements, presumably to view objects in front of the head, followed almost immediately by similarly large divergent ones; other short-interval saccades occur immediately after large saccades and appear to be correction saccades.

\section{Magnitude of saccades}

Although the medians of the magnitude of the horizontal components of the saccades in the two species are quite similar (eagle, $2.8^{\circ}$, frogmouth, $2.7^{\circ}$ ), the shape of the magnitude distributions is quite different (Fig. 6). In the frogmouth, it is prominently skewed, with a long tail toward the large magnitudes, whereas in the eagle the distribution is more nearly Gaussian. In the unrestrained rabbit, the distribution is more like that of the eagle (Collewijn, 1977). This difference in the shape of the distributions results in the mean amplitude of the frogmouth saccades being nearly twice that of the eagle $\left(4.9^{\circ}\right.$ versus $\left.2.9^{\circ}\right)$. In both species, many of the smallest saccades occur accompanying a rather large saccade in the other eye, as though the animal's oculomotor system is, for the moment, in the service of that eye.

\section{Direction of saccades}

One of the most dramatic differences between the oculomotor behavior of the two species is that during most eagle saccades (73\%), both eyes move in the same direction, whereas during most frogmouth saccades $(63 \%)$, the eyes move in opposite directions (Fig. 7). In this respect, the eagle's eye movements were rather more like that of a monkey or human, whereas the frogmouth was very different indeed. For this analysis, we excluded the saccades with amplitudes of less than $1^{\circ}$, to eliminate errors in assessing the direction of very small saccades.

\section{Relation between saccadic amplitude and change in vergence}

Although most of the eagle's saccades are in the same direction, few are strictly conjugate (Fig. 8). Instead, at all saccade amplitudes,

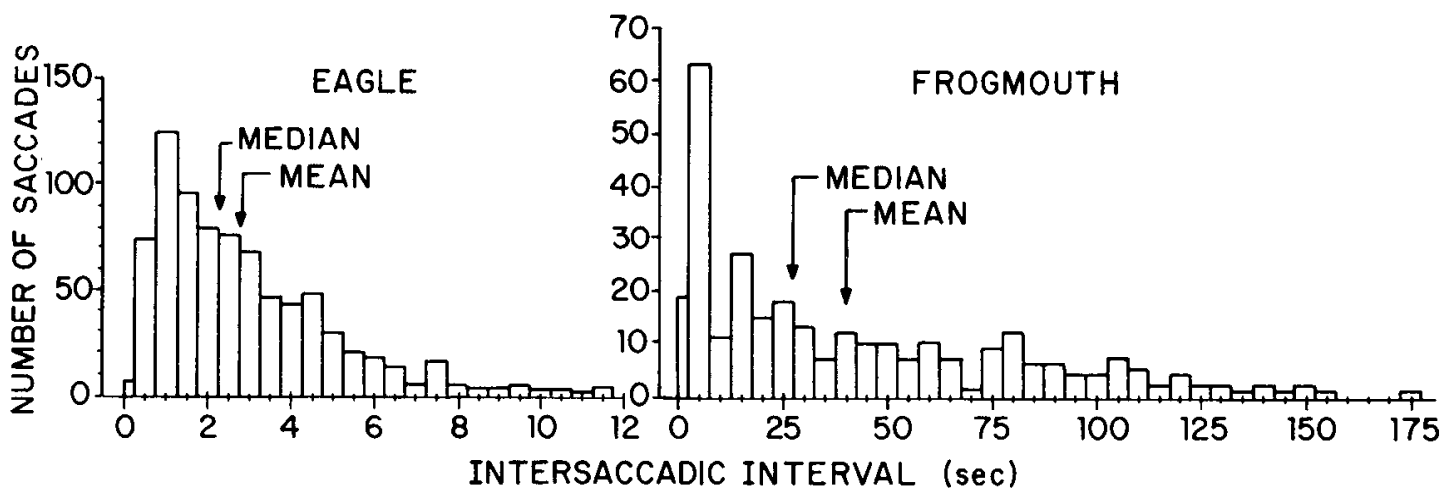

Figure 5. Interval between spontaneous saccades. The eagle makes saccades more than 10 times as frequently as the frogmouth. The peak in the frogmouth distribution arises largely from brief excursions of the eyes into the frontal visual field. 


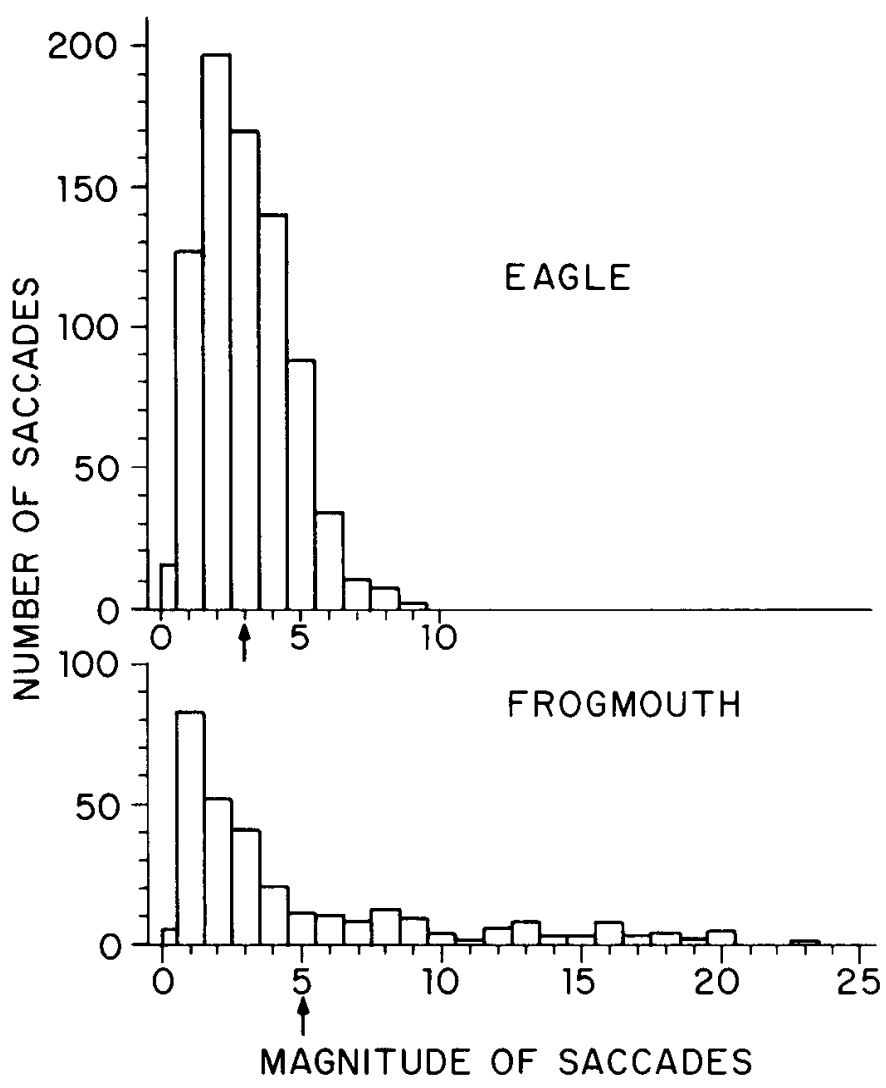

Figure 6. Distribution of magnitude of horizontal component of saccades (in degrees). Although the medians of the distributions are similar, the frogmouth makes many more large saccades, resulting in a difference in the means (arrows).

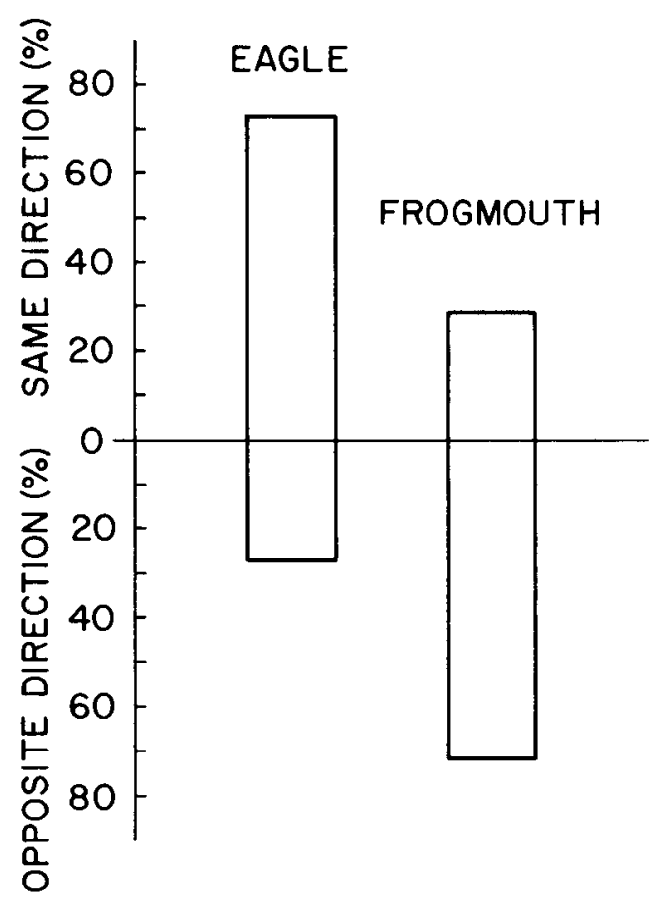

Relative saccade direction in 2 eyes

Figure 7. Percentage of saccades in which the two eyes move in the same direction or in opposite directions. In the eagle, most saccades are in the same direction; in the frogmouth, most are in opposite directions. a range of vergence changes is seen, centered on $0^{\circ}$ (conjugate saccades). In the frogmouth, on the other hand, two entirely different patterns are seen depending on the amplitude of the eye movement. The small amplitude $\left(<4^{\circ}\right)$ eye movements of the frogmouth resemble those of the eagle. In the case of larger amplitude movements, however, in every single case the eyes move in opposite directions, thereby giving the largest possible vergence change. These eye movements occur when the eyes leave their diverged state near the primary position to briefly view something in front of the animal, presumably with the binocular visual field. These large changes in vergence are generally followed shortly thereafter by a similarly large divergent movement back to the region of the primary position.

\section{Observations of other types of eye movements}

1. We found that in some situations the frogmouth does not display a vestibulo-ocular reflex (VOR). While we cannot say for certain whether the VOR is suppressed or is always absent, the apparent ubiquity of the VOR among vertebrates (including other birds; Wallman et al., 1982) argues for suppression as the more likely explanation. One such situation arose in the course of calibrating the eye coils; we found that rotating the frogmouth as briskly as possible by hand through an angle of $10^{\circ}$ or $20^{\circ}$ in dim light produced no apparent compensatory eye movement. Specifically, by doing a series of 20 such $10^{\circ}$ rotations of the frogmouth clockwise and counterclockwise and plotting the two distributions of eye positions immediately after each type of rotation, we found the change in eye position to be consistently within the error of measurement of the ophthalmoscopic method $\left( \pm 1^{\circ}\right)$.

It appears that this suppression is specific to the VOR rather than reflecting an absence of all compensatory reflexes. If a frogmouth is rotated with the head unrestrained, a vestibulocollic reflex with a gain near one is evoked. If a bird is in the same head-restrained situation described above but with a patterned screen moved around it at 5 to $10^{\circ} / \mathrm{sec}$, strong optokinetic nystagmus results.

2. Optokinetic nystagmus (OKN) is directionally asymmetric. In both species, stimulation of OKN by a moving belt of patterned material surrounding the animal produced substantially stronger OKN in the temporal-to-nasal direction than in the other direction (Fig. 3f), a pattern found in general in afoveate species with lateral eyes and with predominantly crossed optic nerves (Collewijn, 1969; Tauber and Atkin, 1968). The frogmouth and eagle differed, however, in that the mean eye position during OKN was deviated in the direction of the slow phases (i.e., to the left for a leftward moving stimulus) in the frogmouth but was deviated in the quick phase direction in the eagle. Thus, in this regard, the eagle is like monkeys or humans, which also deviate to the quick-phase side during OKN, and the frogmouth is more like rats, chickens, and rabbits, which deviate toward the slow-phase side.

\section{Discussion}

\section{Differences between frogmouth and eagle}

We have described several differences in the oculomotor behavior of frogniouth and eagle that can plausibly be related to the different emphasis placed on binocular vision as opposed to panoramic vision in the two species. To summarize briefly, the frogmouth holds its foveas substantially diverged (Fig. 4) and makes infrequent saccades (Fig. 5), which tend to move the eyes in opposite directions (Fig. 7). Many of its eye movements are very large (Fig. 6) and convergent (Fig. 8), briefly creating a large binocular visual field in front of the animal. The eagle, on the other hand, keeps its foveas more frontally placed, so that they are mostly within the binocular visual field. It makes frequent saccades, which tend to be small and in the same direction in both eyes, if not conjugate.

Thus, the eagle's behavior emphasizes foveal, binocular vision at the expense of panoramic vision, as would seem appropriate for an animal hunting from great heights and lacking predators. In contrast, the frogmouth maximizes panoramic vision most of the time and 


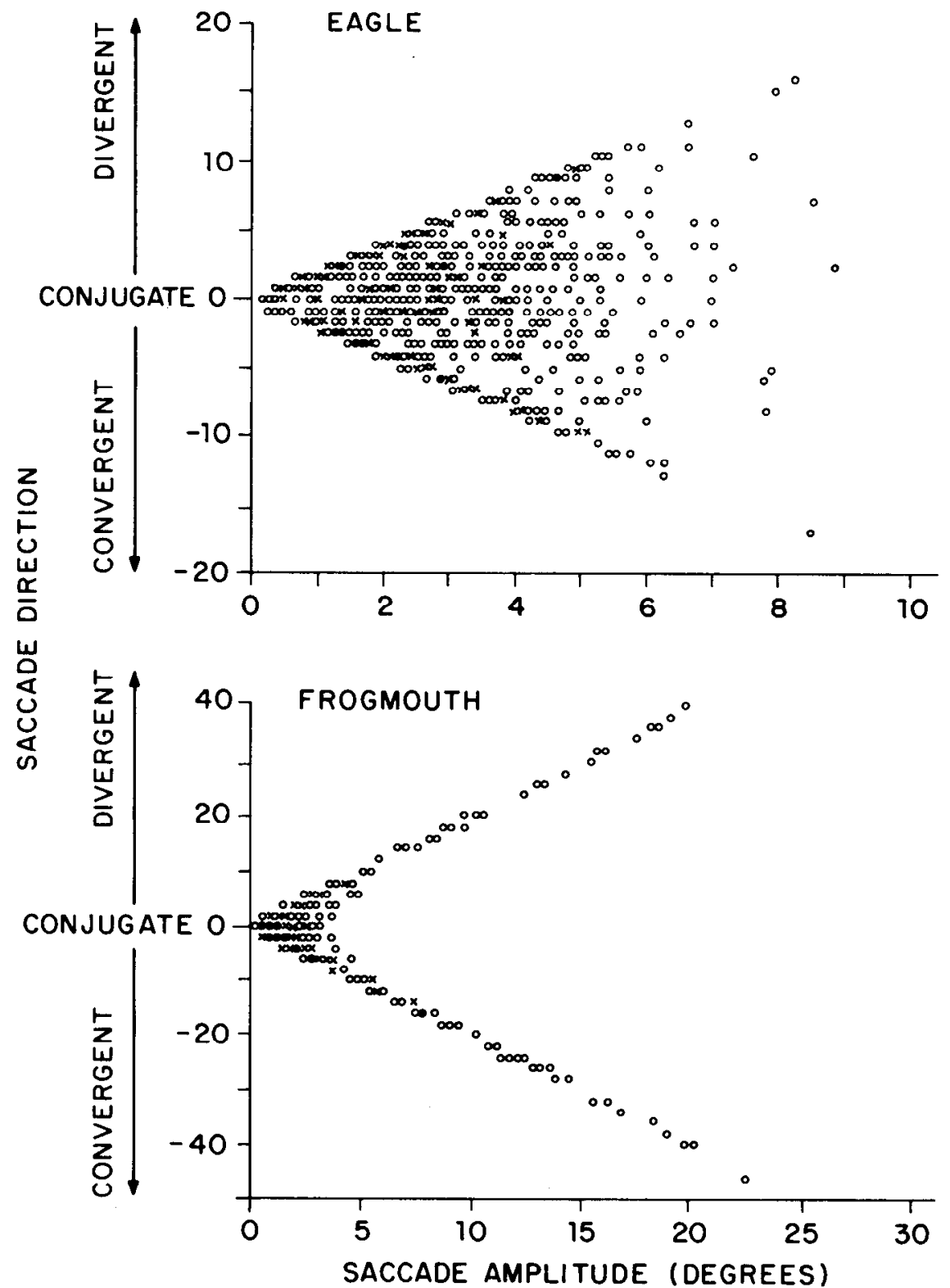

Figure 8. Change in vergence resulting from saccades as a function of average horizontal saccade amplitude of the two eyes. Each symbol represents either one or two saccades (open circles), 3 to 5 saccades (crosses), or six or more saccades (solid circles). Among saccades of less than $5^{\circ}$, both species show a even distribution ranging from saccades that are maximally convergent or divergent (the change in vergence equals twice the average saccade amplitude) to those that are conjugate. The frogmouth, in addition, makes larger amplitude saccades that are all maximally convergent or divergent.

only briefly converges to gain a binocular view of objects. This behavior seems appropriate for an animal for whom panoramic vision would be important to detect both predators and prey, both of which could appear suddenly over a wide angular subtense. In addition, when the frogmouth is in the camouflage posture with its eyes nearly immobile, a wide angle of view would presumably be of selective advantage. The frogmouth's very low saccade frequency may be related both to a decreased need for eye movements because of the wide field of view and to a need to avoid compromising the camouflage posture with frequent saccades, particularly in view of its conspicuous yellow irides. In the potoo, Nyctibius, a related Caprimulgiform bird with an identical camouflage strategy, the eyelids have two notches which allow simultaneous forward and backward vision when the eyelids are apposed covering the salient irides (Borrero, 1974). One may be justified in concluding that there is strong selection pressure operating to reduce the conspicuousness of the eyes in the camouflage posture, by minimizing the degree of eye movement or covering the irides or both. Other nonpredatory birds such as the chicken (J. Wallman, unpublished data) or the pigeon (J. D. Pettigrew and B. Frost, unpublished data) have a frequency of saccades comparable to that observed in the eagle.

It should be emphasized that the frogmouth and eagle are not extreme cases of specialization toward panoramic vision on the one hand and toward binocular vision on the other. The fact that the frogmouth's eye has a single fovea which looks forward, rather than laterally as in the pigeon, suggests the importance of binocular vision, as does the frequency of convergent eye movements. In addition, electrophysiological recording from the frogmouth's visual Wulst (the analogue of the striate cortex) has revealed neurons with binocular receptive fields; these neurons would require that the fovea in each eye be directed toward the same target for optimal stimulation (J. D. Pettigrew, unpublished data). Conversely, the eagle is not entirely oriented toward binocular vision, since it has a lateral fovea and, at least under our experimental conditions, does not generally rest with its binocular foveas aligned, but rather with them near the edge of the region of binocular overlap. In contrast, owls possess more extreme specializations for binocular vision: their eyes are 


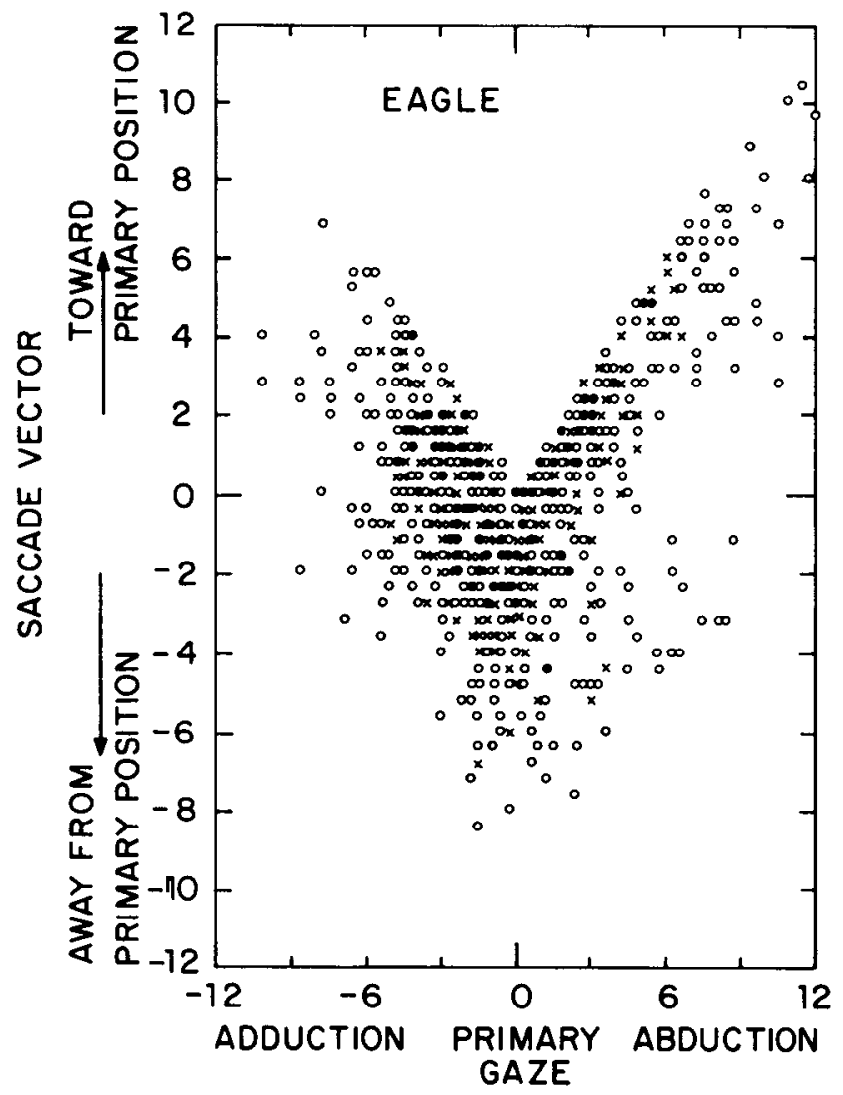

EYE POSITION (deg)

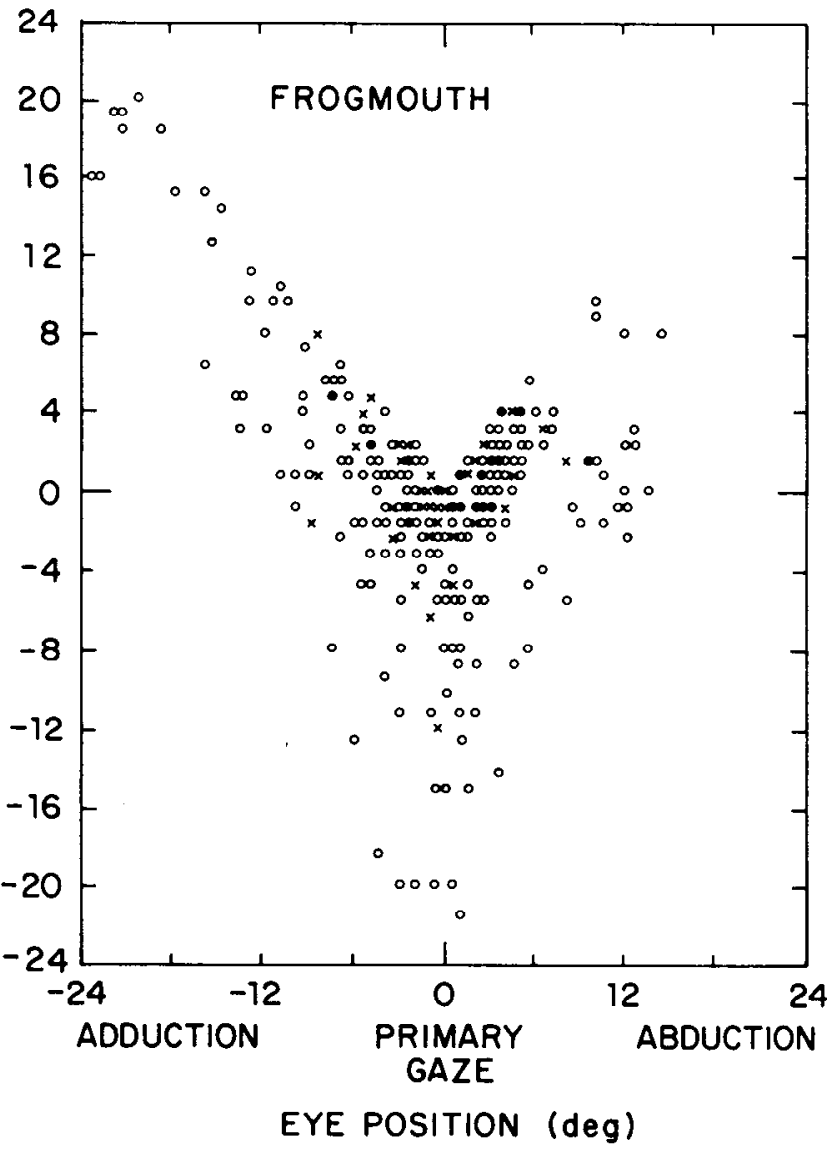

Figure 9. Relationship between size and direction of saccades with respect to the primary position and the eye position at the start of the saccade. The vertical axis plots the net change in distance from the primary gaze position as a result of the saccade. Thus, all the points must fall below the diagonal lines representing saccades that bring the eye exactly to the primary position. The symbols represent one saccade (open circles), two to three saccades (crosses), or four or more saccades (solid circles).

more frontally placed in the head, they have almost no ocular mobility (Steinbach and Money, 1973) and their visual cortical areas contain retinal-disparity-tuned neurons similar to those of highly binocular mammals (Bravo and Pettigrew, 1981; Pettigrew, 1979).

The function of the eagle's having the foveas within the binocular field in the primary position may be related to a mechanism for quickly returning to a state of full binocular fusion with the foveas aligned. It seems plausible that in animals that move the eyes substantially independently, there exists no neural signal to indicate the vergence angle of the eye as has been found in monkeys (Judge and Cumming, 1983; Mays, 1981) and that proprioceptive measures of position are inadequate to permit accurate calculation of saccade trajectories to ensure foveal alignment. Conceivably, if the foveas are within the binocular ficld, binocular ncurons with extremely large retinal disparities may help to program these fovea-aligning saccades.

\section{Primary position of gaze}

The results presented here raise questions concerning the function of the primary position of gaze. For example, why should an animal that makes spontaneous saccades over a range of $38^{\circ}$ spend $90 \%$ of its time with the eyes within less than $7^{\circ}$ of one position, as is the case with the frogmouth?

A number of possibilities exist: (1) minimizing ocular fatigue or energy expenditure; (2) keeping the eye in a position providing the greatest area of visual targets that can be acquired in one saccade; (3) providing the ecologically appropriate balance between frontal and panoramic vision; (4) providing a reference point for calibration of proprioceptive signals from the eye muscles; and (5) facilitating oculomotor or perceptual accuracy of localization by keeping most eye movements within a small area, the calibration of which can be easily maintained.

Regardless of the function of keeping the eyes near the primary gaze, the question remains of how il is achieved. To the best of our knowledge, this has not been studied in any species. Since, in general, the eyes do not drift, whatever mechanism exists must be integrated into the saccade-generating machinery. Several possibilities occur to us. The simplest is a threshold model; when the oculomotor system senses that the eye is more than a certain distance from the primary position, it generates a saccade back to the vicinity of primary gaze. The results of plotting saccade size versus distance from primary position do not show evidence of such a mechanism. A different, and perhaps more plausible, mechanism might bias the direction and amplitude of saccades such that the further from the primary position the eye is, the greater would be the probability that the next saccade will bring the eye toward the primary position; that is, that the distance from primary gaze is a factor summed with others in programming the goal of the next saccade. Such a mechanism would be analogous to a taxis, as used to describe the oriented movements of microorganisms. Figure 9 suggests the existence of such a mechanism. In both species, saccades initiated with the eye away from the primary position show a loose tendency to be of an appropriate magnitude and direction to return to the primary position. Saccades trom the vicinity of the primary position of course move the eye away from the primary position. There is an interesting species difference, however, that depends on which side of the primary position the eyes started. In the frogmouth, there is an especially strict tendency for the eye to return to the primary position when the eye is adducted. This represents the saccades that return the eye from the brief binocular 


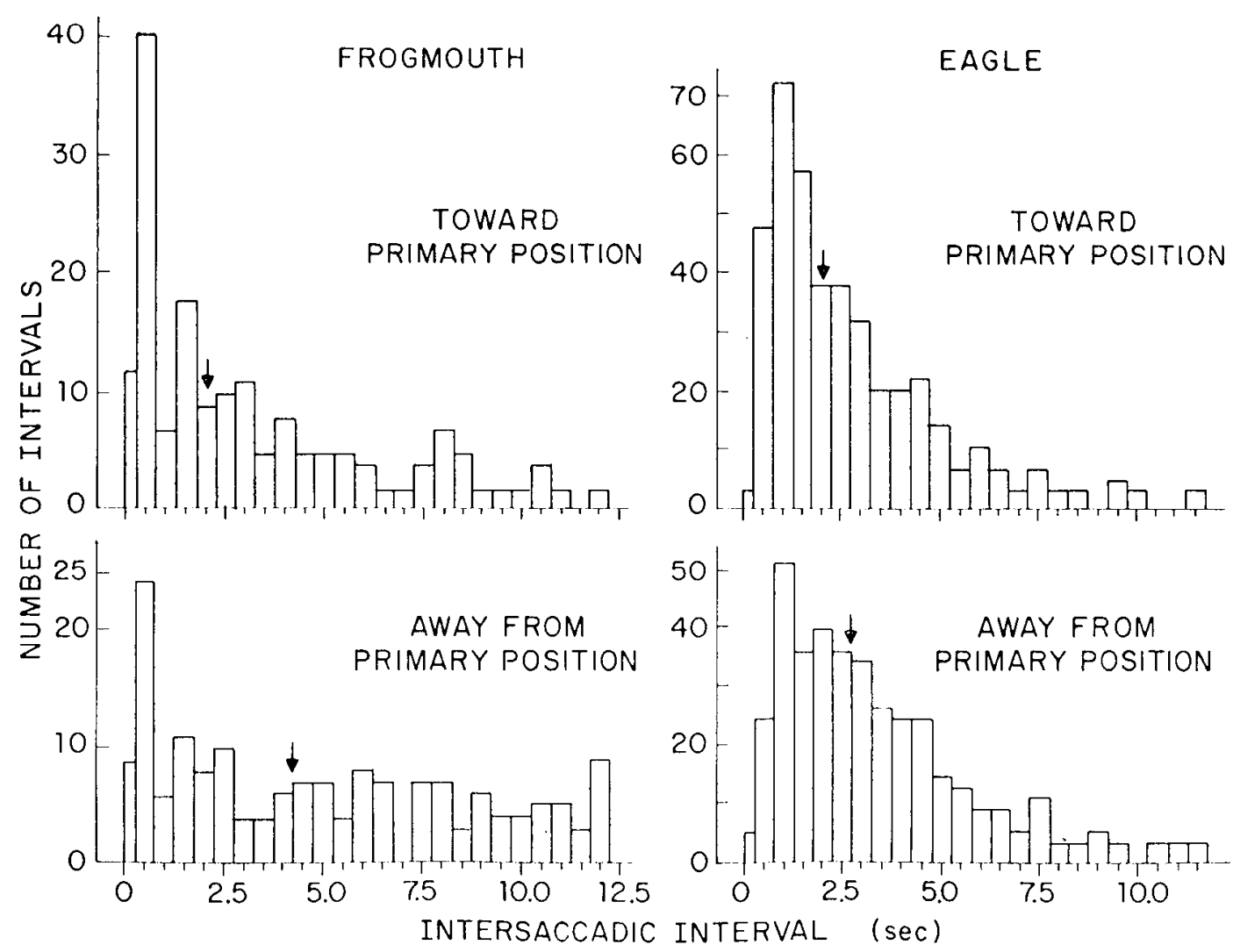

Figure 10. Comparison of the distributions of time intervals preceding those saccades that result in the eye being closer to the primary position and those that result in the eye being further from the primary position. The tendency seen here of saccades toward the primary position occurring after shorter intervals tends to make the eyes return to the vicinity of the primary position. Arrows point to medians.

forays into the frontal field. In contrast, in the eagle, the eyes are more likely to return at once to the primary position when in abduction, perhaps because when the eyes are frontally directed they participate in eye movements more governed by the needs of binocular vision and hence less likely to return at once to the primary position.

Finally, a third possible mechanism for maintaining the primary position is that saccades made in a direction away from the primary position occur after a longer intersaccadic interval then those made in a direction toward the primary position. This mechanism would, by itself, produce a statistical tendency for the eye to spend more time closer to primary gaze; it is analogous to a kinesis, by means of which microorganisms, for example, become more concentrated in well-lit areas by simply being more active in the dark, despite having no ability to orient towards light. Figure 10 shows some evidence for such a mechanism, particularly in the frogmouth. Saccades loward the primary position occur after shorter irltervals than those away from the primary position. These differences are statistically significant (frogmouth: toward primary position, median $=3.77 \mathrm{sec}, n=102$; away from primary position, median $=5.81$ $\mathrm{sec} ; n=110$; eagle: toward primary position, median $=2.55 \mathrm{sec}, n$ $=315$; away from primary position, median $=3.05 \mathrm{sec}, n=307$; in both species $p<0.01$ by Mann-Whitney test). ${ }^{5}$

\section{Binocular yoking}

Despite the greater incidence of approximately conjugate saccades in the eagle compared to the frogmouth, both of these species

\footnotetext{
${ }^{5}$ The first few bins are somewhat misleading since they include the saccades returning the eye to primary position after brief, convergent excursions, presumably to view objects in front of the animal; therefore, in the statistical analysis we have omitted intervals of $1 \mathrm{sec}$ or less. Without this omission, the effect shown here is even stronger.
}

would be regarded as having rather weakly yoked eye movements compared to those of mammals, in which all saccades are quite conjugate. This raises the question of what might be the physiological explan lation of this difference.

In mammals, one accepted mechanism of yoking is at the level of the motoneurons. Internuclear neurons in the vicinity of the abducens nucleus receive the same input as lateral rectus motoneurons and make excitatory connections with medial rectus motoneurons in the contralateral oculomotor nucleus. Indeed, one-third of the abducens neurons are thought to be internuclear neurons (Baker and Spencer, 1981). In chickens as well, there is some evidence of neurons projecting from the abducens nucleus to the contralateral oculomotor nucleus (Wold, 1978); the saccades of chickens are approximately as weakly yoked as are those of the birds studied here (J. Wallman, manuscript in preparation). One could speculate that the degree of conjugacy in both birds and mammals may depend on the extent to which the inputs to the motoneurons also terminate on internucleur neurons. Perhaps in birds fewer internuclear neurons exist than in mammals or they have a less powerful influence on their synaptic targets. Alternatively, perhaps there are both excitatory and inhibitory internuclear neurons in birds, which might help explain not only the weaker and more variable yoking of the eyes, but also the symmetric convergent and divergent eye movements of the frogmouth.

In another respect, the binocular yoking seems equally strong in birds and mammals. In both groups, saccades occur in both eyes essentially simultaneously. This was observed qualitatively in both of the species studied here. In a quantitative study of chicken saccades, we found that all saccades start within $8 \mathrm{msec}$ of the saccade in the fellow eye and $90 \%$ start within $4 \mathrm{msec}$ (J. Wallman and $\mathrm{A}$. McPhun, unpublished results). Since a prevalent view is that pauser neurons initiate saccades, the pausers may have similar binocular connectivity in birds and mammals. 
Other parts of the oculomotor system have yet other patterns of yoking. Avian optokinetic nystagmus, at least in pigeons and chickens, shows moderate yoking, such that an open eye can drive a covered eye with reasonably high gain (Gioanni et al., 1981), but with both eyes open, the gain is lower in the eye receiving nasal-totemporal visual motion. In contrast, the $25-$ to $30-\mathrm{Hz}$ sinusoidal oscillations that accompany each saccade in chickens are quite strictly conjugate, even during saccades in which the the eyes move in opposite directions. The same diversity in premotor patterns of binocular yoking may also exist in mammals, but be obscured by the tight yoking of the eye movements at the level of the motoneurons. These patterns may be better revealed in animals not constrained so strictly to obey Hering's Law.

\section{References}

Baker, R., and R. F. Spencer (1981) Synthesis of horizontal conjugate eye movement signals in the abducens nucleus. Jpn. J. EEG EMG September (Suppl.): $49-59$

Borrero, J. I. (1974) Notes on the structure of the upper eyelid of Potoos (Nyctibius). Condor 76: 210-211.

Bravo, H., and J. D. Pettigrew (1981) The distribution of neurons projecting from the retina and visual cortex to the thalamus and tectum opticum of the barn owl, Tyto alba, and the burrowing owl, Speotyto cunicularia. J. Comp. Neurol. 199: 419-441.

Collewijn, H. (1969) Optokinetic eye movements in the rabbit: Input-output relations. Vision Res. 9: 117-132.

Collewijn. H. (1977) Eye and head movements in freely moving rabbits. J. Physiol. (Lond.) 266: 471-498.

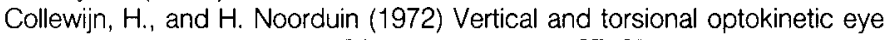
movements in the rabbit. Pflugers Arch. 332: 87-95.

Cramp, S., and K. E. L. Simmons (1981) The Birds of the Western Palearctic, Oxford University Press, Oxford.

Fuchs, A. F., and D. A. Robinson (1966) A method for measuring horizontal and vertical eye movements chronically in the monkey. J. Appl. Physiol. 21: $1068-1070$
Gioanni, H., J. Rey, J. Villalobos, J. J. Bouyer, and Y. Gioanni (1981) Optokinetic nystagmus in the pigeon (Columba livia) I. Study in monocular and binocular vision. Exp. Brain Res. 44: 362-370.

Judge, S. J., and B. Cumming (1983) Brainstem neurons related to accomodation and vergence. Soc. Neurosci. Abstr. 9: 1087.

Judge, S. J., B. J. Richmond, and F. C. Chu (1980) Implantation of magnetic search coils for measurement of eye position: An improved method. Vision Res. 20: 535-538.

Lanchester, B. S., and R. F. Mark (1975) Pursuit and prediction in the tracking of moving food by a teleost fish (Acanthaluteres spilomelanurus). J. Exp. Biol, 63: 627-645.

Mays, L. E. (1981) Neuronal correlates of vergence eye movements. Soc. Neurosci. Abstr. 7: 133.

Nye, P. W. (1969) The monocular eye movements of the pigeon. Vision Res. 9: $133-144$

Pettigrew, J. D. (1979) Binocular visual processing in the owl's telencephalon. Proc. R. Soc. Lond. Biol. 204: 435-454.

Schodde, R., and I. J. Mason (1981) The Nocturnal Birds of Australia, Lansdowne Editions, Melbourne.

Steinbach, M. J., and K. E. Money (1973) Eye movements of the owl. Vision Res. 13: 889-891.

Tauber, E. S., and A. Atkin (1968) Optomotor responses to monocular stimulation: Relation to visual system organization. Science 160: 13651367.

Turkel, J., and J. Wallman (1977) Oscillatory eye movements with possible visual function in birds. Soc. Neurosci. Abstr. 3: 158.

Wallman, J., and J. Velez (1985) Directional asymmetries of optokinetic nystagmus: Developmental changes and relation to the accessory optic system and to the vestibular system. J. Neurosci. 5: 317-329.

Wallman, J., J. Velez, B. Weinstein, and A. E. Green (1982) Avian vestibuloocular reflex: Adaptive plasticity and developmental changes. J. Neurophysiol. 48: 952-967.

Wold, J. E. (1978) The vestibular nuclei in the domestic hen (Gallus domesticus) III. Ascending projections to the mesencephalic eye motor nuclei. J. Comp. Neurol. 179: 393-406.

Wood, C. A. (1917) The Fundus Oculi of Birds Especially as Viewed by the Ophthalmoscope, pp. 1-180, The Lakeside Press, Chicago. 